\title{
SUMMARY: THE GALACTIC BACKGROUND RADIATION *
}

\author{
J. L. Puget \\ Radioastronomie, Ecole Normale Supérieure \\ 24 , rue Lhomond \\ 75231 Paris Cedex 05, France
}

\begin{abstract}
The most striking aspect of this conference with respect to galactic studies has been the confrontation between wavelengths. The study of the background radiation requires absolute measurements of low brightness over very extended regions. Such measurements are known to be extremely difficult. We now can confront data on the galactic background at all frequencies. The data from far ultraviolet to submillimeter give particularly useful constraints on both the direct interpretation of the data and on their astrophysical interpretation.
\end{abstract}

\section{DIRECT STARLIGHT AND THE INTERSTELLAR RADIATION FIELD}

The interstellar radiation field (ISRF) in the solar vicinity is defined as the radiation intensity received from outside of the solar system integrated over all directions. This radiation is observed with foreground emission of scattered solar light and infrared radiation reemitted by dust particles inside the solar system (the zodiacal light component of the background radiation). This radiation can be recognized by the dependence of its intensity with ecliptic coordinates. It can also be recognized by specific features from the solar spectrum for the scattered part and by the spectral energy distribution for the reemitted part. The ISRF can be separated into two parts: direct starlight and diffuse galactic light which is the scattered and reemitted starlight by the interstellar particles. The integrated direct starlight is not dominated by very faint numerous stars but by stars between 9th and 14th magnitude depending on the wavelength and the galactic latitude (Witt, this volume). It is thus possible to measure the diffuse galactic radiation on lines of sight where no stars are detectable. The ISRF is dominated by direct star light from $912 \AA$ to $5 \mu \mathrm{m}$. The diffuse radiation is only a few percent at most wavelengths except in the visible where it reaches $20 \%$ (Toller, this volume).

In a simple model of stars and dust uniformly distributed in a disc, the luminosity of the galactic disc at a given frequency $v L_{v}$ (all quantities are given per logarithmic frequency inter$\mathrm{val}$ ) is related to the average brightness at the pole $v B_{0 v}$ and to the integrated direct starlight $v u_{v}$

$$
\begin{gathered}
v u_{v}=G(v) v L_{v} / c, \\
v u_{v}=2 G(v) 4 \pi v B_{0 v} / c .
\end{gathered}
$$

$G(v)$ is a geometrical factor which can be approximated by:

$$
G(v)=1 / 2\left[1-\ln \left(\sin b_{\min }\right)\right]
$$

\footnotetext{
* All references in this summary are from Proc. IAU 139: Galactic and Extragalactic Background Radiation, ed. S. Bowyer and Ch. Leinert (Dordrecht: Kluwer Academic Publishers).
} 
where $b_{\min }$ is the latitude at which the optical depth $\tau_{v}$ reaches one

$$
\tau_{v}\left(b_{\min }\right)=1 \text {. }
$$

$G(v)$ is 1.2 in the far UV, 1.75 in the visible, and 2 in the near infrared. In the far infrared, $G(v)$ reaches about 5 because the bright galactic ridge from the inner galaxy contributes significantly to the integrated flux. The ISRF is shown in Figure 1 in the form of a brightness averaged over all directions. There are very few serious discrepancies between star counts and galactic models on the one hand and photometric measurements of the integrated background radiation on the other hand. The brightness at high galactic latitude in the blue and UV is somewhat less than expected (Toller, Gondhalekar, this volume). This is equivalent to saying that in relation (2), $G(v)$ does not decrease in the ultraviolet as predicted by the simple model and remains around 2. The reason is the nonuniform distribution of $\mathrm{O}$ and $\mathrm{B}$ stars which are concentrated in the Gould's belt.

One still unresolved discrepancy is the excess of $2.4 \mu \mathrm{m}$ emission observed in the inner galaxy in a thin disc associated with the molecular ring and active star formation (Fazio, this volume).

\section{DIFFUSE GALACTIC LIGHT: SCATTERED AND REEMITTED RADIATION}

What are we trying to learn from the study of the galactic diffuse radiation? One motivation is not galactic physics but cosmology (see Longair, this volume). We want to know how good the "cosmological windows" are. These windows are both spatial and spectral windows and we need to be able to evaluate the intensity and spectrum of the remaining galactic contribution in the spots at high galactic latitude where the diffuse galactic radiation reaches a minimum. For galactic physics we want to find out the amount and properties of dust in the various phases of the interstellar medium and put constraints on the topology and filling factors of these various phases.

The average extinction properties of the local interstellar medium are relatively well known through observations of stars. Difficulties arise when one tries to separate the scattering part from the absorbing one in the extinction. There have been many local studies of scattering of light in the visible and UV. The main difficulty in the interpretation of such data is due to the unknown geometrical factors which do not allow an independent determination of the albedo and of the scattering phase function of the dust nor a proper absolute ratio between scattered and incident radiation. The photometric surveys carried aboard satellites that cover a large fraction of the sky allow a systematic and statistically significant correlation between scattered radiation and the column density of interstellar gas, allowing a quantitative comparison of scattered radiation vs. starlight. The comparison with the column density of interstellar gas can be most easily done using $21 \mathrm{~cm}$ surveys.

An important new element for the understanding of the properties of dust appeared with the IRAS data which give an all sky survey of the reemitted radiation in the mid and far infrared. The tight correlation observed at high latitude between the $100 \mu \mathrm{m}$ brightness and $\mathrm{HI}$ column density (Boulanger, this volume) shows that the dust in all HI clouds absorbs roughly the same amount of energy (within a factor of two). The fraction of the high latitude emission following a cosecant law (and thus associated with galactic disc emission) presents the same ratio to HI column density as that observed for individual clouds. This implies that most of the absorbing dust at high latitude is associated with $\mathrm{HI}$ clouds. 
The correlation between HI column density and scattered radiation (especially in the UV) is not as good as the correlation with the far infrared emission. This is probably related to well-known variations of the extinction curve in the UV. The study of the variations of these dust properties can in fact be done better by a direct comparison between the $100 \mu \mathrm{m}$ emission and the scattered light (Guhathakurta, this volume). The IRAS data have shown that this reemitted radiation did not have the energy distribution predicted by the standard dust models. A very large excess is present in the mid infrared (the $12 \mu \mathrm{m}$ and $25 \mu \mathrm{m}$ bands of IRAS) over that expected from standard dust models. This is best interpreted by the introduction of very small particles transiently heated by single photons, the smallest of which are probably large polycyclic aromatic hydrocarbons molecules (PAHs). The ratio of photometric emission at 12 and $100 \mu \mathrm{m}$ is, as a first approximation, a measure of the abundance of the very small particles.

The ionized component is traced by $\mathrm{H} \alpha$ emission and by dispersion measures of pulsars. Both types of measurements give the same picture. The column density at the galactic pole is about $10^{20} \mathrm{~cm}^{-2}$ (about $1 / 3$ of the column density of atomic hydrogen). There is not yet a definitive identification of scattered or emitted dust emission from this component. However, recent studies of the far UV background presented by Hurwitz et al. (this volume) and C. Martin et al. (this volume) are indicative of the dust scattering in directions where $N_{H I}$ approaches zero. The intensity of this scattered light is consistent with the hypothesis that it arises from dust associated with a column density of $10^{20} \mathrm{~cm}^{-2}$ and standard dust-gas ratios (Hurwitz et al., this volume). As already mentioned, the whole high latitude galactic emission identified by its $\operatorname{cosec}(b)$ dependence is completely accounted for by the emission of $\mathrm{HI}$ clouds and that suggests dust depletion in the ionized component. This question cannot be settled before we know how much of the $\mathrm{H} \alpha$ emission is correlated with the $\mathrm{HI}$ emission (Munch, this volume).

Figure 1 summarizes the data presented on the diffuse galactic light in the form of an average spectrum at the galactic pole. It includes only data from extensive surveys for which a correlation with neutral hydrogen column density has been carried out. The values plotted use these correlation data and normalize the intensity to either an HI column density of $3.10^{20} \mathrm{~cm}^{-2}$ or an $100 \mu \mathrm{m}$ intensity of $2.5 \mathrm{MJy} / \mathrm{sr}$. These values are shown as open squares. The emission from galactic dust includes the emission at $3.3 \mu \mathrm{m}$ feature from PAHs extrapolated from the data of Giard (this volume), 12, 25, 60, and $100 \mu \mathrm{m}$ from IRAS (Boulanger, this volume), 102, 137 and $262 \mu \mathrm{m}$ from rocket data (Matsumoto, this volume). At $3.3 \mu \mathrm{m}$ the open square shows the energy averaged over a frequency range $\delta \ln v=1$; the feature is also represented assuming a width $\delta v / v=0.05$. The scattered part relies on satellite data in the ultraviolet given by Jacobsen, de Vries, and Paresce (1987), and Lequeux (this volume) and from Pioneer in the visible (Toller, this conference). Although the number of wavelengths at which scattered radiation has been measured over most of the sky is quite limited, it appears quite clearly that the ratio between the ISRF and the scattered radiation is maximum at visible wavelengths. A decrease when going from visible to the near IR is expected because of the maximum size of the particles making the bulk of interstellar dust. The decrease in the ultraviolet is more interesting because it probably indicates a decrease of the albedo. Determinations of the albedo in the ultraviolet give widely varying answers between very low values of 0.2 to 0.3 like those obtained by Hurwitz et al. (this volume) or values as high as 0.7 as illustrated by the measurements reported by Witt (this volume). For data averaged over most of the sky, the geometrical factors should be well averaged and the results more reliable than those obtained by observations of particular regions.

A related question is that of the energy budget of interstellar dust. Martin Harwitt in his historical introductory talk reminded us that we have believed in energy conservation since 


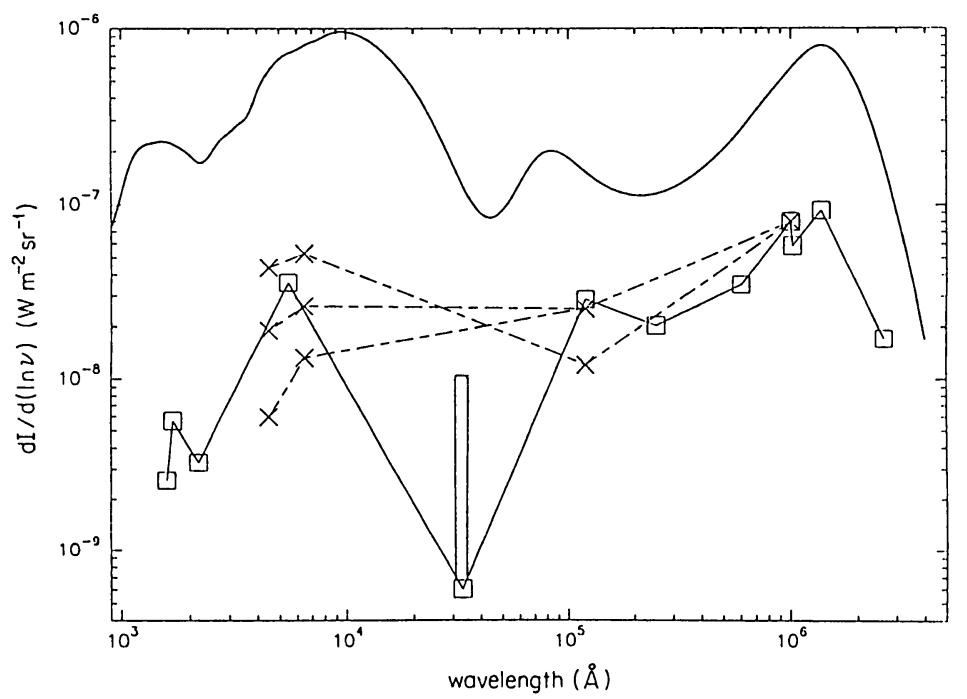

Figure 1. The solid line shows the interstellar radiation field averaged over all directions. The bump around $7.7 \mu \mathrm{m}$ is an evaluation of the contribution of the PAHs features based on the $12 \mu \mathrm{m}$ IRAS band and the $3.3 \mu \mathrm{m}$ measurements. The open squares show the averaged scattered and emitted radiation at the galactic pole (see text for references), corresponding to an HI column density of $3.10^{20} \mathrm{~cm}^{-2}$. At $3.3 \mu \mathrm{m}$ both the energy in a frequency interval $\delta \ln v=1 ; \square$ and a representation of the line are given. The crosses connected by dashed lines show the scattered and emitted radiation for three individual clouds normalized to the same $100 \mu \mathrm{m}$ flux (from Guhathakurta, this volume).

1845. In the particular case of interstellar dust the IRAS data tell us that the energy reradiated in the infrared is $7.10^{-31} \mathrm{~W}$ per $\mathrm{H}$ atom. How is this energy accounted for? Absorbed stellar radiation is the only possible source for this infrared radiation, which accounts for about one third of the energy output of the Galaxy (Mezger, this volume). For the averaged interstellar radiation field as plotted in Figure 1, this puts constraints on the dust properties and generally speaking it excludes models with high albedos. This is in agreement with the prediction for a rather low albedo by dust models including PAHs and very small grains (below 0.5 at all wavelengths falling to 0.3 in the far ultraviolet).

The general correlation found in extensive surveys between HI column density and scattered and reemitted radiation should not hide the fact underlined by many speakers that these correlations do not hold on the small scale where many deviations from the average relations are found (Verschuur, Lequeux, Boulanger, Schnur and Mattila; all in this volume). This point is illustrated in Figure 1 by the data of Guhathakurta for three clouds. They are shown by the crosses connected by dashed lines after normalisation to the same $100 \mu \mathrm{m}$ intensity of 2.5 MJy/sr. They find that the scattered light in the blue can vary by a factor of 6 . The color of the observed radiation between blue and red is redder than expected from pure scattering and the red excess is probably due to red fluorescence. Futhermore this red excess might be correlated with the relative amount of $12 \mu \mathrm{m}$ emission attributed to the very small grains. These variations are probably associated with the same physical processes as the large variations of the IRAS colors observed in and at the edges of molecular clouds (Boulanger, this volume). 


\section{THE COSMOLOGICAL WINDOWS}

When the zodiacal component is included, the minima in the foreground emission are:

- in the ultraviolet (both zodiacal and diffuse galactic radiation decrease rapidly with decreasing wavelength).

- the near infrared with a minimum around $3 \mu \mathrm{m}$ as long as regions of very low galactic emission are chosen; if not the $3.3 \mu \mathrm{m}$ band should be avoided.

- the submillimeter at wavelengths longer than $300 \mu \mathrm{m}$ where the galactic diffuse emission is decreasing fast.

- in the mid infrared, when the galactic background is rather weak (wavelengths shorter than $50 \mu \mathrm{m}$ ), the zodiacal component is getting very strong but it is quite smooth and hence small-scale observations might be feasible.

In terms of spatial distribution, the plot of Figure 1 corresponds to the average emission above latitude $50^{\circ}$. The statistics of the distribution of the $100 \mu \mathrm{m}$ emission show that about $1 \%$ of the sky has a brightness which is less than one third of this average. A few spots present only an upper limit at about one third of this value.

\section{QUESTIONS}

This confrontation between wavelengths raised a number of interesting questions. I have made a list of some of them.

- The albedo is still not well established especially in the far ultraviolet; is it decreasing with frequency in the far ultraviolet?

- Are the local variations in color of both the scattered and the reemitted radiation correlated? Are those variations connected with the variations of the extinction curve?

- Does this reflect changes in the size spectrum of interstellar dust particles (especially the smallest ones)?

- Is the dust depleted in the diffuse ionized gas seen in $H \alpha$ ?

- Same question for the hotter phases of the interstellar medium like the one seen by its CVI emission.

Much progress should be expected from detailed comparisons of the infrared emission with the hotter phases of the interstellar medium. The stucture seen in the $\mathrm{H} \alpha$ emission should allow such work. The COBE data should be most effective in that respect because of the capability of COBE to do absolute measurements with an excellent brightness sensitivity. On smaller scales ISO, AELITA and SIRTF should also improve these comparisons. Nevertheless in both the infrared (with the missions mentioned above) and the ultraviolet (with experiments and projects like FAUST, HUBE, FUVIS, and PAX) progress will come from the combination of good sensitivity and the use of spectral information which allows a nonambiguous separation of the various background components. The importance of the spectral data is stressed by Harwit (this volume) and a first illustration was given by Bowyer (this volume).

In the optical, the progress is clearly coming from the use of new large two-dimensional detectors giving at the same time both structural and photometric data. The use of the spatial fluctuations will certainly also become a useful tool for the separation of the various components following the work done on the infrared cirrus.

\section{REFERENCES}

Jacobsen, P., de Vries, J. S., and Paresce, F. 1987, Astron. Astrophys. 183, 335. 